\title{
Topographic Paracentral Corneal Thickness With Pentacam and Orbscan: Effect of Acoustic Factor
}

\author{
Javier González-Pérez, O.D., M.Sc., Ph.D., Jose M. González-Méijome, o.D., Ph.D., María T. Rodríguez Ares, M.D., Ph.D., \\ and Manuel A. Parafita, M.D., Ph.D.
}

Purpose: To evaluate the effect of an acoustic factor (AF) on the comparison of central corneal thickness (CCT) and peripheral corneal thickness (PCT) measurements with Orbscan II and Pentacam.

Methods: The CCT and PCT at 1,2, and 3 radial distances from the corneal apex were measured using Orbscan II and Pentacam in 22 right eyes of 22 healthy adults ( 7 men, 15 women). Three measures were obtained from each 1 of the 25 points measured and then compared to gauge the agreement between both devices at the corneal center and anular areas located at 1-, 2-, and 3-mm distances from the central measurement. Orbscan II readings were considered with and without an $\mathrm{AF}$ correction.

Results: Pentacam provides statistically significant higher values than Orbscan II does at all the 25 locations analyzed $(P<0.001)$. With a few exceptions, the average difference was fairly constant between 20 and $40 \mu \mathrm{m}$ for all the corneal locations. A high correlation existed between central readings $\left(r^{2}=0.927 ; P<0.001\right)$ and average thickness at each one of the annular areas being analyzed $\left(r^{2}=0.897\right.$ at $1 \mathrm{~mm}, r^{2}=0.876$ at $2 \mathrm{~mm}$, and $r^{2}=0.870$ at $\left.3 \mathrm{~mm}\right)$; Pentacam minus Orbscan II value averaged for all the points changed from $-28 \pm 10$ to $-22 \pm 9 \mu \mathrm{m}$ after the removal of the AF in Orbscan II.

Conclusions: Central and peripheral measurements of the corneal thickness obtained with Orbscan II and Pentacam are significantly different. The removal of the $\mathrm{AF}$ in Orbscan II renders lower mean differences but decreases the agreement between both systems and potentially induces an overestimation of CCT and PCT by Orbsan II compared with Pentacam.

Key Words: Pachometry—Peripheral corneal thickness-PentacamOrbscan II-Bland-Altman.

(Eye \& Contact Lens 2011;37: 348-353)

$\mathrm{C}$ entral corneal thickness (CCT) measurement is a critical procedure in many clinical conditions, including the diagnosis and follow-up of corneal disease, presurgical and postsurgical evaluation, and assessment of corneal physiology. However, in several aspects of clinical practice and ophthalmic research,

From the Ocular Surface and Contact Lens Research Laboratory (J.G.-P., J.M.G.-M., M.A.P.); Department of Applied Physics (Optics) (J.G.-P.), University of Santiago de Compostela, A Coruña, Spain; Clinical and Experimental Optometry Research Laboratory (J.M.G.-M), Center of Physics (Optometry), School of Sciences, University of Minho, Braga, Portugal; and Department of Surgery (Ophthalmology) (M.T.R.A., M.A.P.), University of Santiago de Compostela, A Coruña, Spain.

Supported by national grant PI081380 from Instituto de Salud Carlos III, Spain granted to Ocular Surface and Contact Lens Research Group GI-1750.

The authors have no funding or conflicts of interest to disclose.

Address correspondence and reprint requests to Javier González-Pérez, O.D., M.Sc., Ph.D., Department of Applied Physics (Optometry), School of Optometry, Campus Vida, Universidade de Santiago de Compostela,

15782-A Coruña, Spain; e-mail: javier.gonzalez@usc.es

Accepted August 10, 2011.

DOI: $10.1097 /$ ICL.0b013e3182323dcf peripheral corneal thickness (PCT) is even more critical to identify peripheral pathologic conditions or peripheral thinning effects that could compromise the outcomes of a refractive surgery procedure.

Although ultrasound (US) pachometry is usually recognized as the gold standard against which all the remaining techniques should be compared and attempts have been designed to map the $\mathrm{PCT}^{1,2}$ and corneal volume ${ }^{3}$ using this technology or new high-frequency US biomicroscopy ${ }^{4}$ modern optical methods have the advantage of evaluating this parameter noninvasively. Orbscan II and Pentacam are 2 devices that obtain the corneal thickness from the central 8-10 mm of the cornea using translational or rotational "slit-scanning principles," respectively. The acoustic factor (AF) was first used in Orbscan II to increase the agreement of CCT measurements of Orbscan with ultrasonic measurements. However, the removal of AF in Orbscan II has shown to improve the agreement with Pentacam for central measurements. ${ }^{5}$ The accuracy of PCT measurements obtained with Orbscan II has been questioned in the literature because of large deviations compared with US measurements. ${ }^{6}$ In that study, Orbscan II largely overestimated PCT when compared with US measures along the vertical meridian. Several studies have compared the agreement of the Orbscan and Pentacam systems for CCT in normal eyes ${ }^{7-12}$ but only rarely considering peripheral corneal data. ${ }^{13,14}$

Considering the relevance of PCT in ophthalmic care, particularly in the surgical environment, it is necessary to explore the values obtained in the peripheral cornea with Pentacam compared with Orbscan II that has been already subjected to comparison with other standards. Although a direct comparison between Pentacam and US in the periphery might render more definitive results, the topographical measurement of PCT numerous points has proved to be feasible but time consuming and less convenient in clinical practice. ${ }^{1,2,15}$

To our knowledge, there are not many reports about the direct comparison between Pentacam and Orbscan in the periphery of the cornea. ${ }^{14}$ As such, we were trying to explore what the relationship was and how it could change removing the AF. To improve the knowledge about the agreement of different optical devices in measuring PCT, slit-scanning Orbscan II and Pentacam rotational Scheimpflug tomography systems were compared not only at the central location but also at 24 peripheral locations at 1,2 , and $3 \mathrm{~mm}$ along 8 different semimeridians of normal corneas.

\section{METHODS}

\section{Subjects}

Twenty-two healthy subjects ( 7 men, 15 women), with ages ranging from 20 to 29 years $(21 \pm 2)$, were recruited from the Universitywide population. Inclusion criteria required that the 
subjects did not suffer from any ocular condition or injury at the moment of the study. None of the subjects exhibited corneal pathologic conditions or corneal scarring, or had previously been submitted to corneal refractive surgery, nor were they using any ocular or systemic medication. Only 3 subjects reported using soft contact lenses occasionally, but none used them during the 3 days before thickness measurements.

Sample size was calculated based on the average values of CCT obtained with Pentacam pachometry to warrant a power of $90 \%$ with a statistical significant level of 0.05 , so 21 eyes were necessary. After explaining the nature of the experimental procedures, informed consent was obtained from each subject before data acquisition. All the procedures followed the guidelines of the Declaration of Helsinki and were reviewed and approved by the Scientific and ethics Committee of the University of Santiago de Compostela, Spain.

\section{Instruments and Measurements}

Corneal thickness was measured with Orbscan II (Bausch \& Lomb, Rochester, NY) and Pentacam (Oculus, Wetzlar, Germany). All the instruments were calibrated before each measurement session using the test provided by the manufacturer. To minimize the effect of diurnal corneal hydration changes on pachometry data, all the measurements were performed in the afternoon between 14:00 and 18:00. ${ }^{16}$

Measurements were recorded at 3 different radial distances of 1 , 2 , and $3 \mathrm{~mm}$ from the corneal center along 8 semimeridians located at $45^{\circ}$ intervals $\left(0^{\circ}, 45^{\circ}, 90^{\circ}, 135^{\circ}, 180^{\circ}, 225^{\circ}, 270^{\circ}\right.$, and $\left.315^{\circ}\right)$. Each patient was measured three times, and the three readings at each location were averaged. The eight measurements within each annular area were averaged to obtain an estimation of the "average thickness" at each peripheral region. Orbscan II readings were computed both with and without the $\mathrm{AF}$ of 0.92 as recommended by the manufacturer.

\section{Statistical Analyses}

Data were analyzed using the statistical package SPSS version 18.0. Normal distribution of variables was assessed using the Kolmogorov-Smirnov test. Correlations between measurements obtained with different instruments were obtained. The $95 \%$ limits of agreement (LoAs $=$ mean difference between both instruments $\pm 1.96 \mathrm{SD}$ of the differences) were also calculated for each pair of instruments according to previously described methodology. ${ }^{17}$ Orbscan II data have been extracted with and without the AFs, and both sets of data were compared with Pentacam data in a separate analysis. Then, to simplify the analysis and data presentation, all data in each chord diameter $(2,4$, and $6 \mathrm{~mm})$ have been averaged. The level of significance was established at $\alpha=0.05$. PCT data were averaged for each radial distance (i.e., 1-mm ring, 2-mm ring, and $3-\mathrm{mm}$ ring as the result of 8 measures averaged from each ring, respectively).

\section{RESULTS}

In Table 1 mean value, SD, and the value of statistical significance for topographical corneal thickness differences are presented separately for each technique. Point-by-point analysis demonstrated that Orbscan II readings are lower on average for all the measured points when the AF is considered and were higher
TABLE 1. Descriptive Statistics of the Difference Between Corneal Thickness Orbscan II Minus Pentacam (Mean \pm SD) With and Without AF Correction for 25 Different Corneal Locations Within the Central $6 \mathrm{~mm}$ of the Cornea ${ }^{a}$

\begin{tabular}{lrcrrr}
\hline & \multicolumn{2}{c}{ No AF } & & AF \\
\cline { 2 - 3 } \cline { 5 - 6 } Orbscan-Pentacam & Mean $\pm \mathrm{SD}$ & $P^{b}$ & & Mean $\pm \mathrm{SD}$ & \multicolumn{1}{c}{$P^{b}$} \\
\hline Central & $+18 \pm 14$ & 0.130 & $-29 \pm 12$ & 0.011 \\
Superior 1 mm & $+21 \pm 17$ & 0.078 & $-27 \pm 14$ & 0.017 \\
Superior 2 mm & $+27 \pm 28$ & 0.036 & $-25 \pm 27$ & 0.044 \\
Superior 3 mm & $+8 \pm 16$ & 0.512 & $-48 \pm 15$ & $<0.001$ \\
Inferior 1 mm & $+28 \pm 30$ & 0.017 & $-19 \pm 29$ & 0.091 \\
Inferior 2 mm & $+32 \pm 16$ & 0.004 & $-18 \pm 15$ & 0.077 \\
Inferior 3 mm & $+34 \pm 21$ & 0.008 & $-20 \pm 22$ & 0.105 \\
Nasal 1 mm & $+28 \pm 17$ & 0.021 & $-20 \pm 15$ & 0.080 \\
Nasal 2 mm & $+35 \pm 17$ & 0.015 & $-17 \pm 17$ & 0.213 \\
Nasal 3 mm & $+32 \pm 23$ & 0.022 & $-23 \pm 24$ & 0.093 \\
Temporal 1 mm & $+11 \pm 14$ & 0.305 & $-35 \pm 12$ & 0.002 \\
Temporal 2 mm & $+27 \pm 33$ & 0.032 & $-21 \pm 32$ & 0.081 \\
Temporal 3 mm & $+19 \pm 30$ & 0.109 & $-32 \pm 29$ & 0.007 \\
Sup-Nas 1 mm & $+22 \pm 17$ & 0.071 & $-26 \pm 14$ & 0.025 \\
Sup-Nas 2 mm & $+33 \pm 15$ & 0.010 & $-19 \pm 13$ & 0.117 \\
Sup-Nas 3 mm & $+12 \pm 23$ & 0.321 & $-43 \pm 22$ & 0.001 \\
Inf-Temp 1 mm & $+16 \pm 11$ & 0.134 & $-30 \pm 10$ & 0.006 \\
Inf-Temp 2 mm & $+27 \pm 11$ & 0.010 & $-21 \pm 11$ & 0.032 \\
Inf-Temp 3 mm & $+28 \pm 15$ & 0.006 & $-23 \pm 16$ & 0.019 \\
Sup-Temp 1 mm & $+13 \pm 17$ & 0.243 & $-33 \pm 14$ & 0.004 \\
Sup-Temp 2 mm & $+13 \pm 22$ & 0.251 & $-36 \pm 20$ & 0.002 \\
Sup-Temp 3 mm & $+1 \pm 25$ & 0.957 & $-52 \pm 24$ & $<0.001$ \\
Inf-Nas 1 mm & $+20 \pm 15$ & 0.086 & $-27 \pm 13$ & 0.016 \\
Inf-Nas 2 mm & $+32 \pm 26$ & 0.012 & $-19 \pm 25$ & 0.125 \\
Inf-Nas 3 mm & $+14 \pm 33$ & 0.353 & $-41 \pm 30$ & 0.005 \\
\hline & & & & \\
\hline
\end{tabular}

${ }^{a}$ Values are expressed in microns.

${ }^{b}$ Paired samples test.

$\mathrm{AF}$, acoustic factor.

than those of Pentacam when the AF is removed. Such differences are apparently higher for the most extreme locations being measured (i.e., 3-mm ring). Table 2 shows the mean differences between both systems, upper and lower LOAs and amplitude of the $95 \%$ confidence interval, with and without AF to obtain Orbscan II measurements. A statistically significant difference $(P<0.01)$ was found between Pentacam and Orbscan systems in CCT with $(29 \pm 12 \mu \mathrm{m})$ and without $(-18 \pm 14 \mu \mathrm{m})$ AF. However, the agreement between the systems was good in both central and peripheral cornea, with a correlation of 0.963 , indicating that differences in CT measurements between the systems was small compared with intersubject variability observed for each instrument.

Figure 1 plots the values of CT obtained at the center and those averaged across the 1-, 2-, and 3-mm rings with (Figs. 2A-D) and without AF (Figs. 2E-H). The correlation was stronger at the center $\left(r^{2}=0.927 ; \quad P<0.001\right)$ and slightly weaker in the periphery $\left(r^{2}=0.897, r^{2}=0.876, r^{2}=0.870\right.$ for $1-, 2-$, and $3-\mathrm{mm}$ areas; $P<0.001)$ when the AF is considered. The same correlations are present after the removal of the AF, although the regression line approaches the 1:1 relationship. Regarding LOAs presented in Figure 2, the confidence was similar for the center and peripheral areas varying from $45 \mu \mathrm{m}$ at the center to $51 \mu \mathrm{m}$ at the $3-\mathrm{mm}$ ring area. Moreover, the differences between instruments seem to be independent of the value being measured for the most peripheral locations $\left(r^{2} \leq 0.304\right)$ when the AF is considered. More peripheral readings obtained at $3 \mathrm{~mm}$ show the opposite trend, but this was not statistically significant. 
TABLE 2. Comparisons Among Pairs of Instruments, Average Difference, Upper and Lower 95\% Confidence Limits, and Statistical Significance With and Without Acoustic Factor in Orbscan II

\begin{tabular}{|c|c|c|c|c|c|c|c|}
\hline Parameter & Acoustic Factor & Mean Diff. Pentacam-Orbscan & Upper Limit & Lower Limit & Range & Significance or Diff. $(P)^{b}$ & Correlation $(P)^{c}$ \\
\hline \multirow[t]{2}{*}{ Center } & On & $29 \pm 12$ & 51.3 & 6.1 & 45.2 & $<0.001$ & 0.963 \\
\hline & Off & $-18 \pm 14$ & 9.7 & -45 & 54.7 & $<0.001$ & 0.963 \\
\hline \multirow[t]{2}{*}{ 1-mm Ring } & On & $27 \pm 13$ & 51.6 & 2.5 & 49.1 & $<0.001$ & 0.947 \\
\hline & Off & $-20 \pm 15$ & 8.7 & -48.7 & - & $<0.001$ & 0.963 \\
\hline \multirow[t]{2}{*}{ 2-mm Ring } & On & $22 \pm 12$ & 46.3 & -2.7 & 49 & $<0.001$ & 0.939 \\
\hline & Off & $-28 \pm 14$ & -1.7 & -54.7 & 53 & $<0.001$ & 0.963 \\
\hline \multirow[t]{2}{*}{ 3-mm Ring } & On & $35 \pm 13$ & 60.9 & 9.2 & 51.8 & $<0.001$ & 0.933 \\
\hline & Off & $-18 \pm 13$ & 7.9 & -44.7 & 52.6 & $<0.001$ & 0.963 \\
\hline
\end{tabular}

${ }^{a}$ Values are in microns.

${ }^{b}$ Statistically significant differences between instruments with the paired samples test.

'Statistically significant correlations (Pearson correlation).

FIG. 1. Correlation analysis between Pentacam and Orbscan II measurements for central readings, average thickness in the 1-, 2-, and 3-mm areas with (A-D) and without Orbscan II acoustic factor (E-H), respectively. Dashed lines represent a 1:1 relationship.
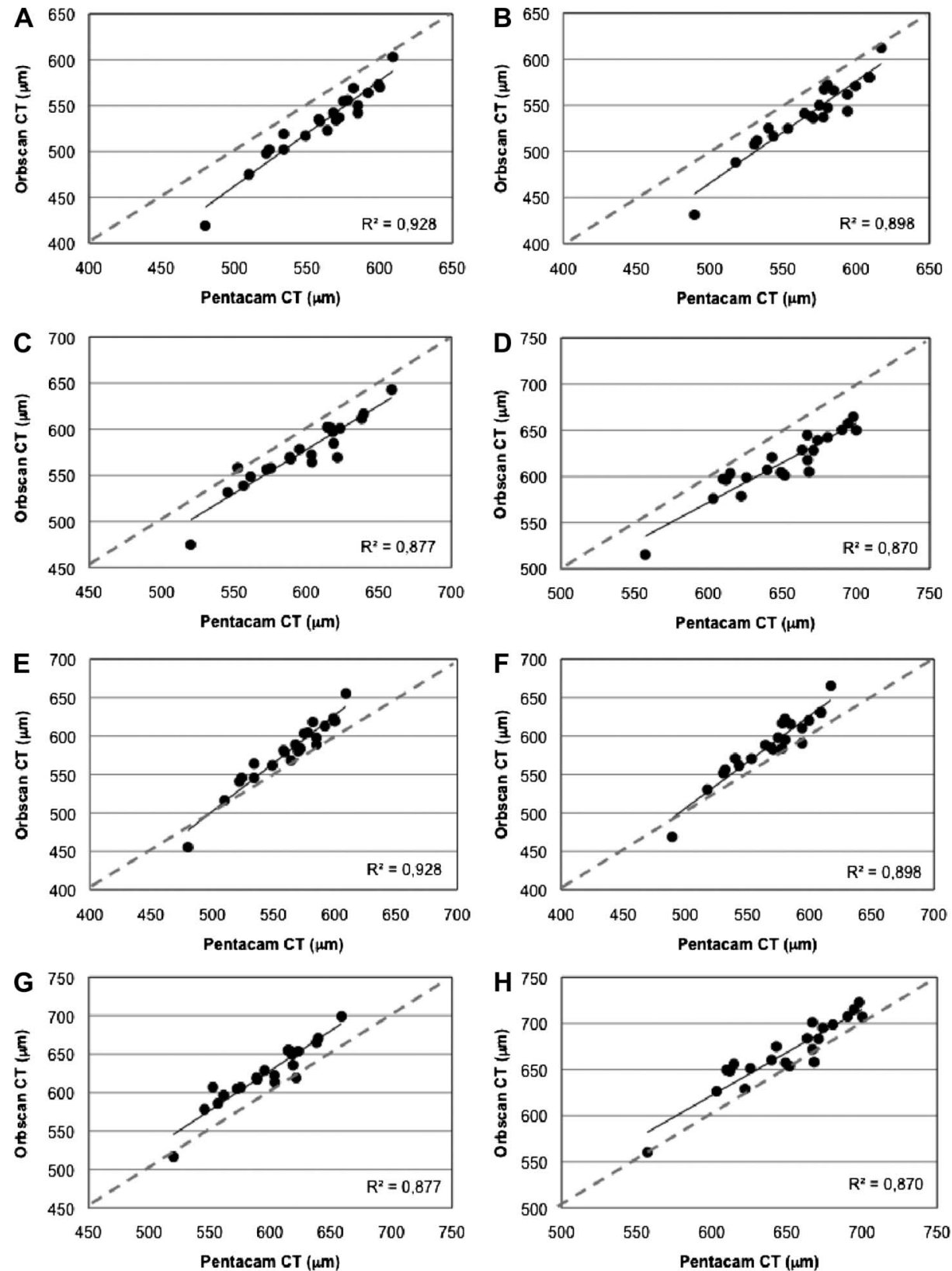
FIG. 2. Bland-Altman views and confidence intervals for comparison between Pentacam and Orbscan II measurements for central readings, average thickness in the 1-, 2-, and 3-mm areas with (A-D) and without Orbscan II acoustic factor $(E-H)$, respectively.
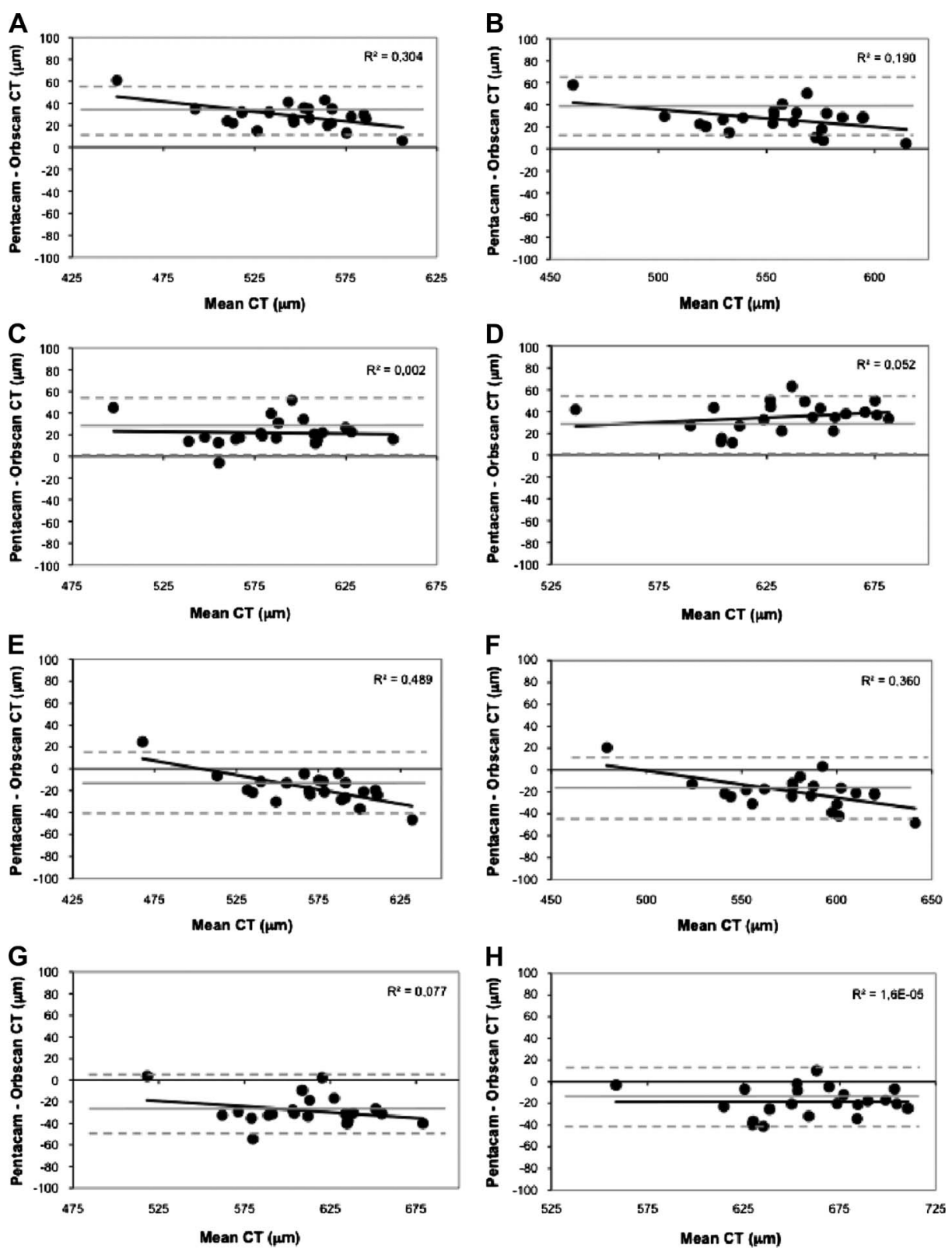

\section{DISCUSSION}

To the best of the authors' knowledge, this is the first time that Orbscan II and Pentacam pachymetry data have been compared for agreement involving 1 central and 24 peripheral locations at 1,2 , and $3 \mathrm{~mm}$ along 8 different semimeridians of normal corneas.

A knowledge of PCT is crucial in many clinical applications; however, this information has only been available in commercial instruments for the last decade. Several studies have shown that PCT measured with noninvasive methods showed a poor agreement with US measures, particularly at the corneal periphery. ${ }^{6}$ The present results have shown that Pentacam and Orbscan II provide significantly different values of thickness across the central $6 \mathrm{~mm}$ in normal corneas. This could be because of the different principles of measurement with the Orbscan II using translation of a slit-scanning device along with the estimation of the anterior corneal surface curvature with a Placido disc, whereas Pentacam relies exclusively on the use of Scheimpflug principle to capture sections of the cornea with a rotary system. The Scheimpflug principle seems to show sharper images of the central and peripheral cornea compared with those of Orbscan, thus justifying different outcomes when measuring PCT. Differences in the algorithms of measurement and edge detection could explain the differences encountered.

Previous studies have compared Orbscan II (using the acoustic adjustment factor recommended by the manufacturer) and Pentacam CCT, but only 2 studies have evaluated PCT at several locations in normal eyes. Lackner et al. ${ }^{7}$ reported that Orbscan II and Pentacam measurements differ from each other such that Pentacam values were thicker and with less variability than Orbscan II values. Both methods showed a similar repeatability. Amano 
et al. ${ }^{18}$ found no significant differences in CCT between Orbscan II and Pentacam, but they noted that Orbscan II tended to underestimate CCT relative to Pentacam for thickness of less than $500 \mu \mathrm{m}$ and tended to overestimate thicknesses of more than 550 $\mu \mathrm{m}$. Both systems were highly repeatable, but Orbscan II had the largest intraexaminer variability. Buehl et al. ${ }^{13}$ concluded that Pentacam and Orbscan II showed small differences in CCT, with an acceptable LoA and larger differences in PCT (measured in 4 points at $1.5 \mathrm{~mm}$ from the center). Hashemi and Mehravaran ${ }^{19}$ found that Orbscan II showed lower CCT values than did Pentacam and concluded that both these systems should not be used interchangeably. Kim et al. ${ }^{20}$ found no significant differences in CCT between Orbscan II and Pentacam, but they noted that Orbscan II tended to underestimate CCT relative to Pentacam for a thickness of less than $450 \mu \mathrm{m}$. Rosa et al. ${ }^{21}$ reported significantly thinner CCT values when measured with the Orbscan II versus when using the Pentacam system in the subjects screened for refractive surgery. Bourges et al. ${ }^{14}$ found a statistically significant difference between Orbscan and Pentacam CCT and PCT with a high agreement between both instruments in relation to CCT values. Pentacam and Orbscan II readings were not found to be interchangeable. Despite significant differences between both systems, a high correlation has been found, even for peripheral areas. In 2003, Gonzalez-Méijome et al. $^{6}$ found a poor agreement between Orbscan II and US pachometry at 1,2 , and $3 \mathrm{~mm}$ from the center in the superior and inferior regions of the vertical meridian. This suggests that PCT measurements with Pentacam, if highly correlated with Orbscan II, would be expected to have a similar relationship with US pachometry. Bourges et al. ${ }^{14}$ suggest that this is in fact the case. These authors found a poor agreement between Pentacam and US pachometry at 1,2, and $3 \mathrm{~mm}$ from the center, which fits well in the correlations found in this study among peripheral locations with both instruments and the already demonstrated lack of comparability between Orbscan II and US pachometry, particularly for the most peripheral locations measured. ${ }^{6}$

Results also showed that when AF is used in Orbscan II, clinicians must expect significantly lower values of corneal thickness compared with Pentacam at all measured locations. Conversely, when AF is removed, Orbscan II reports significantly higher values of CT at all locations compared with Pentacam. Our results showed that the range between upper and lower LoAs increases when the AF is removed. Regarding the clinical implications, Pentacam being a more reliable method than Orbscan, ${ }^{5}$ the AF is necessary to prevent the overestimation of the CT.

Regional differences between different peripheral corneal locations (nasal, temporal, superior, inferior) remain after applying or removing AF as expected because the same factor is applied to all points. Perhaps regional-specific corrections factors would allow the new wave of noninvasive optical instruments to increase their agreement with US technology that still remains the gold standard. This has been indeed suggested by previous authors for the vertical meridian of the cornea. ${ }^{6}$

This study has several limitations, namely, the absence of a gold standard reference such as US pachometry data. The sample size was calculated to capture the differences in CT greater than $20 \mu \mathrm{m}$ that would be enough to compare both methods regarding most of the potential clinical applications. Regarding standard reference measurements, although US pachometry has been used to measure as many as 25 points over the cornea, ${ }^{1-3}$ these approaches are not currently used in clinical applications. Several studies have shown that Pentacam has the best agreement with US pachometry for CCT measurements compared with Orbscan II, so Pentacam could replace US as the standard of comparison for CCT. Despite this, we cannot ensure that Pentacam can be used as the reference for peripheral locations. For example, the studies conducted with Orbscan II showed a gross overestimation of PCT compared with US measurements along the vertical meridian even when the AF was activated to match US measures of the CCT. ${ }^{6}$ Contrary to our initial thoughts, the present results do not show a different agreement for peripheral points compared with CCT (average differences are on the order of 20-30 for CCT and for the 3 paracentral chord diameters). Indirectly, this suggests that similar differences might be expected between Pentacam and US when measuring PCT. Perhaps new developments in the field of high-frequency US biomicroscopy could help to find accurate standards for PCT readings against which other devices can be compared. A further limitation is the fact that we do not evaluate CT over the whole corneal area. However, with such a detailed evaluation of the central 6-mm corneal area, we will ensure sensitivity in detecting most of the relevant features characteristic of postsurgical corneas, keratoconus. Furthermore, the evaluation of the central $6 \mathrm{~mm}$ has proved to be useful to obtain corneal volume calculations from topography and US pachometry ${ }^{3}$ in good agreement with other newer technologies such as the Pentacam.

\section{REFERENCES}

1. Parafita MA, Gonzalez-Meijome JM, Diaz-Rey JA, et al. Corneal thickness mapping by topographical ultrasonic pachymetry. Int Contact Lens Clin 2000;27:12-21.

2. Parafita M, Yebra-Pimentel E, Giraldez MJ, et al. Further information on the knowledge of topographical corneal thickness. Int Contact Lens Clin 1999 26:128-137.

3. Cervino A, Gonzalez-Meijome JM, Ferrer-Blasco T, et al. Determination of corneal volume from anterior topography and topographic pachymetry: Application to healthy and keratoconic eyes. Ophthalmic Physiol Opt 2009; 29:652-660.

4. Wada I. Ultrasound biomicroscopic corneal thickness measurement for corneal thickness mapping. Jpn J Ophthalmol 1997;41:12-18.

5. González-Pérez J, González-Méijome JM, Rodriguez Ares MT, et al. Central corneal thickness measured with three optical devices and ultrasound pachometry. Eye Contact Lens 2011;37:66-70.

6. Gonzalez-Méijome JM, Cervino A, Yebra-Pimentel E, et al. Central and peripheral corneal thickness measurement with Orbscan II and topographical ultrasound pachymetry. J Cataract Refract Surg 2003;29:125-132.

7. Lackner B, Schmidinger G, Pieh S, et al. Repeatability and reproducibility of central corneal thickness measurement with Pentacam, Orbscan, and ultrasound. Optom Vis Sci 2005;82:892-899.

8. Barkana Y, Gerber Y, Elbaz U, et al. Central corneal thickness measurement with the Pentacam Scheimpflug system, optical low-coherence reflectometry pachymeter, and ultrasound pachymetry. J Cataract Refract Surg 2005;31: 1729-1735.

9. Lam AK, Chen D. Pentacam pachometry: Comparison with non-contact specular microscopy on the central cornea and inter-session repeatability on the peripheral cornea. Clin Exp Optom 2007;90:108-114.

10. O’Donnell C, Maldonado-Codina C. Agreement and repeatability of central thickness measurement in normal corneas using ultrasound pachymetry and the OCULUS Pentacam. Cornea 2005;24:920-924.

11. Ho T, Cheng AC, Rao SK, et al. Central corneal thickness measurements using Orbscan II, Visante, ultrasound, and Pentacam pachymetry after laser in situ keratomileusis for myopia. J Cataract Refract Surg 2007;33:11771182.

12. Shankar H, Taranath D, Santhirathelagan CT, et al. Anterior segment biometry with the Pentacam: Comprehensive assessment of repeatability of automated measurements. J Cataract Refract Surg 2008;34:103-113. 
13. Buehl W, Stojanac D, Sacu S, et al. Comparison of three methods of measuring corneal thickness and anterior chamber depth. Am J Ophthalmol 2006;141:7-12.

14. Bourges JL, Alfonsi N, Laliberte JF, et al. Average 3-dimensional models for the comparison of Orbscan II and Pentacam pachymetry maps in normal corneas. Ophthalmology 2009;116:2064-2071.

15. Owens H, Watters GA. An evaluation of the keratoconic cornea using computerised corneal mapping and ultrasonic measurements of corneal thickness. Ophthalm Physiol Opt 1996;16:115-123.

16. Du Toit R, Vega JA, Fonn D, et al. Diurnal variation of corneal sensitivity and thickness. Cornea 2003;22:205-209.

17. Bland JM, Altman DG. Statistical methods for assessing agreement between two methods of clinical measurement. Lancet 1986;1:307-310.
18. Amano S, Honda N, Amano $\mathrm{Y}$, et al. Comparison of central corneal thickness measurements by rotating Scheimpflug camera, ultrasonic pachymetry, and scanning-slit corneal topography. Ophthalmology 2006;113: 937-941.

19. Hashemi H, Mehravaran S. Central corneal thickness measurement with Pentacam, Orbscan II, and ultrasound devices before and after laser refractive surgery for myopia. J Cataract Refract Surg 2007;33:1701-1707.

20. Kim SW, Byun YJ, Kim EK, et al. Central corneal thickness measurements in unoperated eyes and eyes after PRK for myopia using Pentacam, Orbscan II, and ultrasonic pachymetry. J Refract Surg 2007; 23:888-894.

21. Rosa N, Lanza M, Borrelli M, et al. Comparison of central corneal thickness measured with Orbscan and Pentacam. J Refract Surg 2007;23:895-899. 